\title{
Hierarchical modeling of bycatch rates of sea turtles in the western North Atlantic
}

\author{
Beth Gardner ${ }^{1,3, *}$, Patrick J. Sullivan ${ }^{1}$, Sheryan Epperly ${ }^{2}$, Stephen J. Morreale ${ }^{1}$ \\ ${ }^{1}$ Dept. of Natural Resources, Cornell University, Ithaca, New York 14853, USA \\ ${ }^{2}$ NOAA, National Marine Fisheries Service, Miami, Florida 33149, USA \\ ${ }^{3}$ Present address: US Geological Survey, Patuxent Wildlife Research Center, Laurel, Maryland 20708, USA
}

\begin{abstract}
Previous studies indicate that the locations of the endangered loggerhead Caretta caretta and critically endangered leatherback Dermochelys coriacea sea turtles are influenced by water temperatures, and that incidental catch rates in the pelagic longline fishery vary by region. We present a Bayesian hierarchical model to examine the effects of environmental variables, including water temperature, on the number of sea turtles captured in the US pelagic longline fishery in the western North Atlantic. The modeling structure is highly flexible, utilizes a Bayesian model selection technique, and is fully implemented in the software program WinBUGS. The number of sea turtles captured is modeled as a zero-inflated Poisson distribution and the model incorporates fixed effects to examine region-specific differences in the parameter estimates. Results indicate that water temperature, region, bottom depth, and target species are all significant predictors of the number of loggerhead sea turtles captured. For leatherback sea turtles, the model with only target species had the most posterior model weight, though a re-parameterization of the model indicates that temperature influences the zero-inflation parameter. The relationship between the number of sea turtles captured and the variables of interest all varied by region. This suggests that management decisions aimed at reducing sea turtle bycatch may be more effective if they are spatially explicit.
\end{abstract}

KEY WORDS: Longline fisheries $\cdot$ Bycatch $\cdot$ Sea turtles $\cdot$ Hierarchical models

\section{INTRODUCTION}

The incidental catch of sea turtles, termed bycatch, in the pelagic longline fishery has come to the forefront as an issue for fisheries management (Lewison et al. 2004, Kobayashi \& Polovina 2005). In the US Atlantic pelagic longline fishery, a typical set consists of a single mainline (as long as $50 \mathrm{~km}$ ) with hundreds to thousands of baited hooks hanging vertically. For swordfish Xiphias gladius, the gear is mainly set in the upper $50 \mathrm{~m}$ of the water column, which overlaps greatly with the activities of sea turtles. As loggerhead (Caretta caretta) and leatherback (Dermochelys coriacea) sea turtles encounter a longline, they become caught on the hooks or entangled in the gear. Because both species are protected by the
Endangered Species Act and are listed on the IUCN Redlist (IUCN 2004), and because of the large number of interactions with the longline fishery, understanding the dynamics and causes of these interactions is of great interest (Lewison et al. 2004, Lewison $\&$ Crowder 2007). To address the rate of sea turtle bycatch in the longline fishery, time-area closures, gear modifications and a number of other measures have been implemented (Gilman et al. 2006). Although these regulations have decreased undesirable interactions in selected fisheries (Gilman et al. 2007), sea turtle bycatch continues and more precise measures are warranted.

Sea turtles are poikilotherms and thus their distributions are influenced by temperature; however, loggerhead and leatherback sea turtles can occur in a broad 
range of temperatures. Leatherbacks have been observed active in waters ranging from 0 to $30^{\circ} \mathrm{C}$, most likely due to their enhanced abilities to preserve and generate heat (Friar et al. 1972, Paladino et al. 1990). Similarly, loggerheads inhabit a broad range of temperatures; generally, they are observed active in water temperatures as low as $11^{\circ} \mathrm{C}$ (Epperly et al. 1995). The ability to withstand a wide range of temperatures is likely to contribute to the wide distribution of these species, spanning waters from the tropics to the high temperate latitudes. Nevertheless, most sightings of leatherbacks and loggerheads occur between 7 and $27^{\circ} \mathrm{C}$, depending on season and region (Shoop \& Kenney 1992, Epperly et al. 1995, Coles \& Musick 2000). Coles \& Musick (2000) argue that, despite their capabilities, turtles are constrained by preferred temperature ranges. Additionally, as local temperature conditions vary seasonally, sea turtle distributions shift accordingly.

The observed preferred temperature ranges of sea turtles may relate directly to the observed patterns in the pelagic longline fishery, as bycatch rates for sea turtles are known to be influenced by water temperature (Witzell 1999, NMFS 2001, Watson et al. 2005). In general, the greater the water temperature in which fishing gear is set, the greater the chance of encountering a sea turtle (Watson et al. 2005). However, the pattern of bycatch rates may not be so easily explained. Spatial location, or region, also influences the bycatch rates within the pelagic longline fishery. Notably, the catch rate of sea turtles in the northeast distant waters region (NED) has been shown to be higher than other regions for both loggerhead and leatherback sea turtles (Witzell 1999, NMFS 2001). From 1992 to 1999, a disproportionate number of interactions occurred in the NED region, including $69 \%$ of all loggerhead and $45 \%$ of all leatherback sea turtle interactions observed in the US Atlantic fleet, but only $10 \%$ of the total observed fishing hauls. As a consequence of this additional regional increase in the probability of encountering a turtle, fishing in the NED region was restricted for US fishermen from 2001 to 2003. Thus, allowing the effect of temperature to vary by region in statistical analyses would likely improve models to predict sea turtle interactions.

To date, studies of sea turtle bycatch rates with respect to temperature have used linear and tabular type analyses, often yielding general, but not precise, correlations. For example, studies show that sea turtles tend to spend more time in a certain temperature range, with a diminishing likelihood of occurrence as the temperature increases or decreases (e.g. see Epperly et al. 1995, Polovina et al. 2000). This results in a unimodal shape around the most preferred temperature range, a pattern that would not be well captured by a straight line. To account for this unimodal shape, a quadratic term can easily be included in a statistical model. This term can also be allowed to vary by region.

In addition to including region and temperature (with a quadratic term), many other variables may play a role in capturing sea turtles. The amount of time a set is left in the water could influence whether or not a sea turtle interacts with the line, and thus one might assume that soak time is an important factor to include. Watson et al. (2005) found that soak time was a significant predictor of loggerhead sea turtle catches, but notably, not for leatherback sea turtle catches. Additionally, target species might be important since the bait, hook type, and depth of the set will vary based on the target species (Watson et al. 2005). Studies have shown higher relative densities of sea turtles in shelf waters as opposed to deeper waters offshore (Shoop \& Kenney 1992, James et al. 2006), indicating that bottom depth might be another variable related to sea turtle locations and thus captures. While the US fishery primarily targets swordfish in the NED, the longline fishery throughout the western North Atlantic targets a number of other species including bigeye Thunnus obesus and yellowfin tuna T. albacares. The number of variables that could be included in a statistical model is numerous; however, we must be careful not to overparameterize models. This is particularly true when there is a relatively small amount of data, as is the case with sea turtle captures, which are generally considered a 'rare' event. Here, we will focus on establishing a sensible modeling framework that allows more parameters to be included as more data become available, thus continuing to improve modeling efforts to help reduce sea turtle interactions.

We propose a hierarchical zero-inflated Poisson (ZIP) model to examine the influence of environmental variables on the rates of sea turtle bycatch in the US Atlantic pelagic longline fishery. Although much is known about the influence of water temperature on sea turtle distributions, no previous studies have examined the impact of environmental variables, such as water temperature, as a function of region on sea turtle interactions with respect to the pelagic longline fisheries. Additionally, the capture rates of various regions have been noted as being different, but the effect has not yet been quantified. The aim of this study was to combine environmental and fisheries data into a quantitative model to increase understanding of sea turtle occurrence and improve the ability to predict sea turtle bycatch. The intended objective was to build a more flexible modeling framework that would allow for better management decisions with respect to reducing unwanted sea turtle bycatch within the pelagic longline fishery. 


\section{MATERIALS AND METHODS}

Data. The data used in these analyses are from the NOAA Fisheries Atlantic pelagic longline observer program, managed by the Southeast Fisheries Science Center, Miami Laboratory (Beerkircher et al. 2004). The observer program covers the longline fishery, which operates from south of the equator, and throughout the western North Atlantic to the Grand Banks off Newfoundland, Canada (Fig. 1). The observer program typically has an individual observer on 3 to $5 \%$ of the trips per year and in recent years has increased to a range of 5 to $8 \%$. Each trip contains a number of 'sets', which we will use as the sample unit in this analysis. Trained observers record specific data for each set including number of hooks, water temperature $\left({ }^{\circ} \mathrm{C}\right)$, bottom depth of the sea floor (fathoms), soak time (h), and target species and sea turtle catches, all of which were used in these analyses. For temperature and bottom depth, we used the mean of the recorded values for a set taken at the beginning and end of set- ting and retrieving the line (a total of 4 possible data values). The soak time is the amount of time that the longline is in the water from when the line is set to when it is retrieved. The target species categories included in the analysis are swordfish, tuna, yellowfin tuna, bigeye tuna, and mixed. The mixed category includes sets geared towards more than one target species (it should be noted that swordfish are usually one of the species targeted, L. Beerkircher pers. comm.) and the tuna category includes any tuna species (Thunnus spp.). Other data collected, such as gear characteristics and weather conditions, were not examined in this paper. Each of the continuous variables analyzed (temperature, soak time, and bottom depth) were standardized by subtracting the mean and dividing by the standard deviation.

For statistical and regulatory purposes, NOAA Fisheries has 11 geographic regions classifying fishing effort for the US pelagic longline fishery (Fig. 1) and 4 seasons per year (Beerkircher et al. 2004). For this analysis, we examined 3 different regions: Region 7

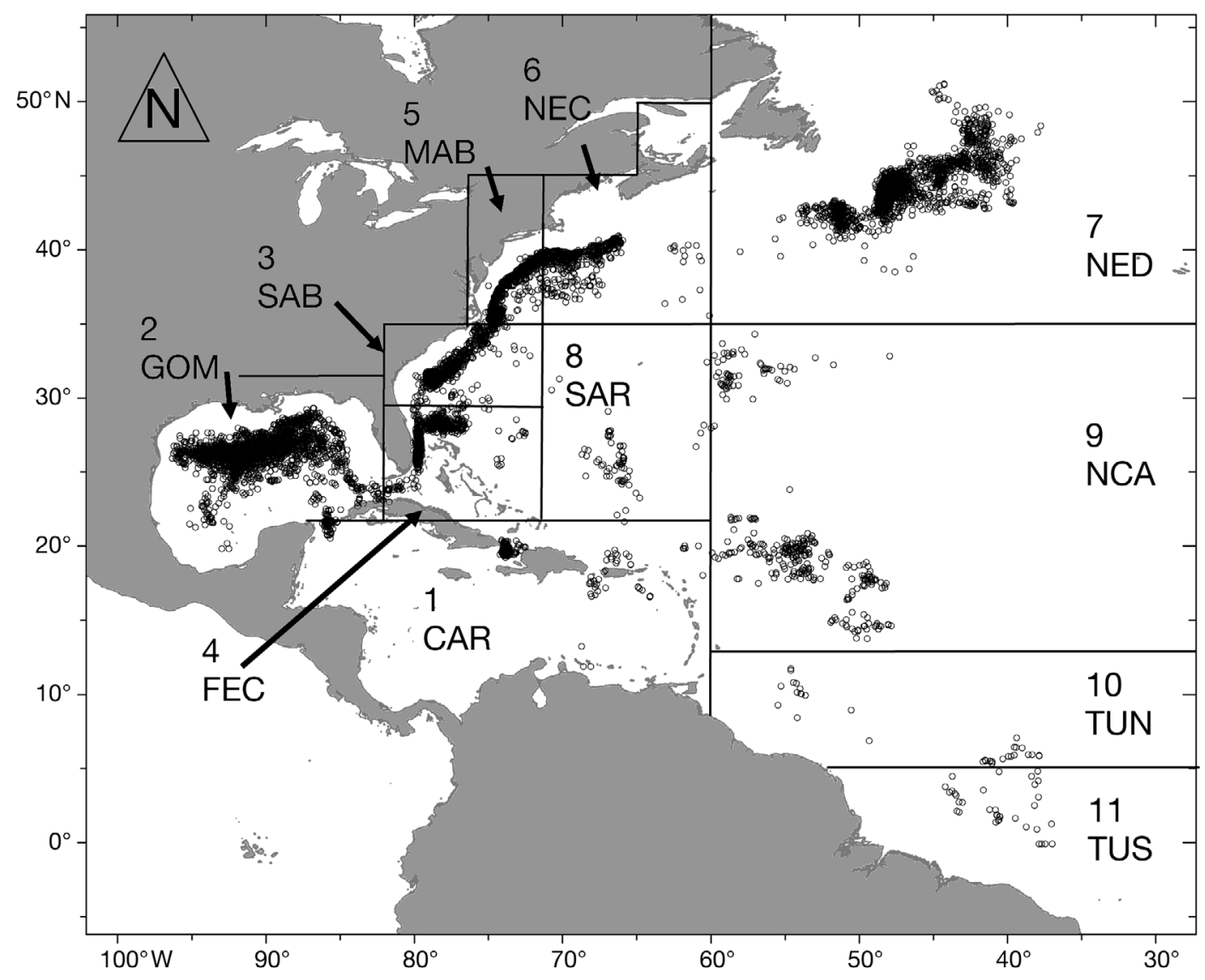

Fig. 1. Geographic regions used to stratify US Atlantic Ocean pelagic longline fishing effort. $1=$ Caribbean $($ CAR), $2=$ Gulf of Mexico (GOM), 3 = South Atlantic Bight (SAB), 4 = Florida East Coast (FEC), 5 = Mid-Atlantic Bight (MAB), $6=$ Northeast Coastal (NEC), 7 = Northeast Distant (NED), 8 = Sargasso Sea (SAR), 9 = North Central Atlantic (NCA), $10=$ Tuna North $($ TUN), and $11=$ Tuna South (TUS). Circles indicate the pelagic longline fishing locations for 1992 to 2003 
(NED); Regions 5 and 6 combined (Northeast Coastal and Mid-Atlantic Bight, termed NECMAB); and Region 2 (Gulf of Mexico, GOM). These regions were selected in order to compare the heavily fished Gulf region with 2 different northern regions. All 3 of these regions have been identified as areas of concentrated loggerhead interactions with the pelagic longline fishery (Williams et al. 1996). To analyze the sea turtle bycatch data, we used the number of turtles captured on each set (there are usually multiple sets that occur during a fishing trip). The data analyzed are from 1992 to 2003. During 2001 to 2003, an experiment to examine gear modifications was conducted in the NED. Because that experiment controlled the fishing and the vessels within the NED, we have excluded those data from the analysis. The model was run separately for the loggerhead and leatherback sea turtles.

The total number of sets used in the analysis was 3866, of which 411 were in the NED, 1449 were in the NECMAB, and 2006 were in the GOM. Additionally, of the total sets, 82 listed the target species as bigeye tuna, 817 as swordfish, 878 as tuna, 832 as yellowfin tuna, and 1257 as mixed. There was a total of 391 loggerhead sea turtles and 374 leatherback sea turtles caught on these observed sets. Of all the sets, 3644 had no loggerhead sea turtle catches while 3577 sets had no leatherback sea turtle catches. For loggerhead sea turtles, there were 150 sets with 1 sea turtle catch, 25 sets with 2 catches, 23 sets with 3 catches, 12 sets with 4 catches, 12 sets with 5 or greater catches with 9 being the highest number caught on 1 set. For leatherback sea turtles, there were 234 sets with 1 turtle catch, 37 sets with 2 catches, 12 sets with 3 catches, 2 sets with 4 catches, and 3 sets with 5 catches, and 1 set with 7 catches.

Model. Exploration of the data indicated that they were not perfectly Poisson distributed (the mean-variance relationship was not upheld), but that the data were over-dispersed for both species. In this case, because of the large number of sets that have no sea turtle catches, zero-inflated (or over-dispersed) models are likely more appropriate for analyzing the data. Here we present a hierarchical approach with a zeroinflated Poisson distribution for the observation model.

First, we describe the captures of sea turtles as having a Poisson distribution with a zero-inflation parameter:

$$
y_{i} \sim \operatorname{Poisson}\left(\lambda_{i} * z_{i}\right)
$$

where $z_{i}$ has a Bernoulli distribution with parameter $\psi$. The $\operatorname{Pr}(z=0)$, i.e. $1-\psi$, is often called the zero-inflation parameter. Under a Poisson regression model we assume that the logarithm of the mean count is related linearly to covariates according to:

$$
\begin{aligned}
\log \left(\lambda_{i}\right) & =\log (\text { hooks })_{i}+\alpha_{r}+\beta_{1, r} * \text { temp }_{i}+\beta_{2, r} * \operatorname{temp}_{i}^{2} \\
& +\beta_{3, r} * \text { depth }_{i}+\beta_{4} * \text { soaktime }+\beta_{5} * \operatorname{target}_{i, k}
\end{aligned}
$$

where $\alpha$ is the intercept, the $\beta$ 's are the parameter coefficients, and $\log ($ hooks) is an offset term (Dobson 1990) equal to the number of hooks (in thousands). Because of the offset term we are effectively looking at rates of bycatch (number of sea turtles per 1000 hooks). The index, $i$, represents a single longline set, $r$ is an indicator for the region in which the longline set occurred (GOM, NECMAB, or NED) and $k$ indicates the target species category (swordfish, tuna, bigeye tuna, or mixed). In order to maintain identifiability in the parameters, we did not code yellowfin tuna as a category, thus it will be included in the intercept term for each region, $\alpha_{r}$, and each of the other target species' parameter estimates will be relative to yellowfin tuna in the results. To incorporate a mechanism for looking at regional differences in parameter values, we specified the coefficients for the intercept, temperature, and temperature squared (the quadratic term for temperature) as separate fixed effects in the model. Any of the coefficients could have been specified as such, but here, we were particularly interested in the regional differences of the mean number of turtles captured and the influence of temperature. The priors for the coefficients are set such that

$$
\beta_{i} \sim \operatorname{Normal}(0,100)
$$

where $\beta$ represents any of the coefficients in the model. Similarly, we specify a non-informative prior for $\alpha$, such that $\alpha_{r} \sim \operatorname{Normal}(0,100)$. Additionally, we used a uniform prior on $[0,1]$ for $\psi$, the prior parameter for $z_{i}$.

Model selection. The posterior model probabilities were computed for each of the possible models (32 total model possibilities). This was done by defining a latent indicator variable, $w_{j}$, for each effect, $j$, of the model (Kuo \& Mallick 1998, Congdon 2007, Royle \& Dorazio 2008). We define the set of latent indicator variables as

$$
w_{j}=\left\{\begin{array}{l}
1 \text { if covariate } j \text { 's coefficient } \neq 0 \\
0 \text { otherwise }
\end{array}\right.
$$

where each $w_{j}$ has a Bernoulli(0.5) prior distribution and multiplies the variables in the model. This prior on $w_{j}$ means that each term has the same chance of being included in the model as it does of not being included in the model, thus the assumption is that all models in the model set are equally probable. We deviate slightly from this assumption in that the squared temperature term includes both $w_{1}$ and $w_{2}$ so that the quadratic term is included in the model only when the linear term is included. There are 32 possible models in the model set. In this case, most models have a prior prob- 
ability of 0.03 (i.e. 1/32), while a few will have a probability of 0.06 (any model where the linear temperature term, $w_{2}$, is 0 will have double the probability). Based on this idea, the full model would be indicated if all $w_{j}=1$ and the null model would be indicated if all $w_{j}=$ 0 . Thus, the prior model weight for the null model is 0.06, while the prior model weight for the full model is 0.03. Using the Markov Chain Monte Carlo (MCMC) histories, we can calculate the posterior model probabilities based on the frequency with which a specific model occurs. Specification of the priors can have an acute influence on the posterior model probabilities (Link \& Barker 2006); as such, one advantage to this model selection technique is the explicit statement of the priors. Changing the priors in this case is very simple and although not done here, one could use this construction to compare the influence of different model priors on the posterior model probabilities.

This formulation of the model can be easily implemented in the software package WinBUGS and the model specification for the data with the model selection parameters is shown in Box 1. The MCMC algorithm was run for 30000 iterations, with 3 chains, the first 10000 were discarded, and posterior summaries were computed from the remaining iterations from the model with the highest posterior weight. Model convergence was checked by running multiple chains in WinBUGS, and by evaluating the potential scale reduction factor (Rhat) in Gelman's (2003) Bugs functions.

Model extension. Because we estimate $z_{i}$ within the model, we can also allow the expected value of $z_{i}, \mathrm{E}\left[z_{i}\right]$, to be a function of one or more of the environmental variables as well. In the section 'Model' above we defined $z_{i}$ as

$$
z_{i} \sim \operatorname{Bernoulli}\left(\psi_{i}\right)
$$

where $\psi_{i}$ had a uniform prior on $[0,1]$. However, we can extend the model and describe $\psi_{i}$ as a function of temperature such that

$$
\operatorname{logit}\left(\psi_{i}\right)=\alpha 1_{r}+\beta_{1, r} * \operatorname{temp}_{i}+\beta_{2, r} * \operatorname{temp}_{i}^{2}
$$

where $b_{1}$ and $b_{2}$ are the parameter coefficients, similar to $\beta_{1}$ and $\beta_{2}$ described above, and $\alpha 1$ is the intercept. Again, $i$ represents a single longline set and $r$ is the indicator for the region (GOM, NECMAB, or NED). This model extension was done to examine the influence of temperature on the likelihood of capturing at least 1 turtle, as compared with the influence of temperature on the number of sea turtles captured. The distinction is subtle but can provide more insights into how variables may influence different model parameters. The other covariates were still included in the model (i.e. soak time, bottom depth, and target species were allowed to influence the mean of the Poisson in the model as described in the section 'Model' above).

\section{RESULTS}

The shape of the distribution pattern of temperatures for all fishing locations is relatively unimodal (mean = 23.81; standard deviation $[\mathrm{SD}]=4.26$ ), with a spike at 24 to $25^{\circ} \mathrm{C}$ (Fig. 2). The total number of turtles caught generally displays a bell-shaped curve across the temperature range. However, when fishing and turtle catches are separated by region, the distributions of temperatures exhibit more detail, with fishing and turtle catches differing by area (Fig. 3). Part of the regional differences may be explained by the observed difference in the range of temperatures for each region (note the peaks in the counts of Fig. 3). These underlying differences are clearly due to varying seasonal conditions at the different latitudes. The fishing loca-

Box 1. WinBUGS specification of the zero-inflated Poisson model for the sea turtle capture data. The model includes the $\mathrm{w}$ terms which are used in the model selection. Note that the squared temperature term has both w1 and w2 so that the quadratic term is included in the model only when the linear term is included. The 'ind' term is used as an indicator of the region and 'target' is an indicator for the target species for particular data point

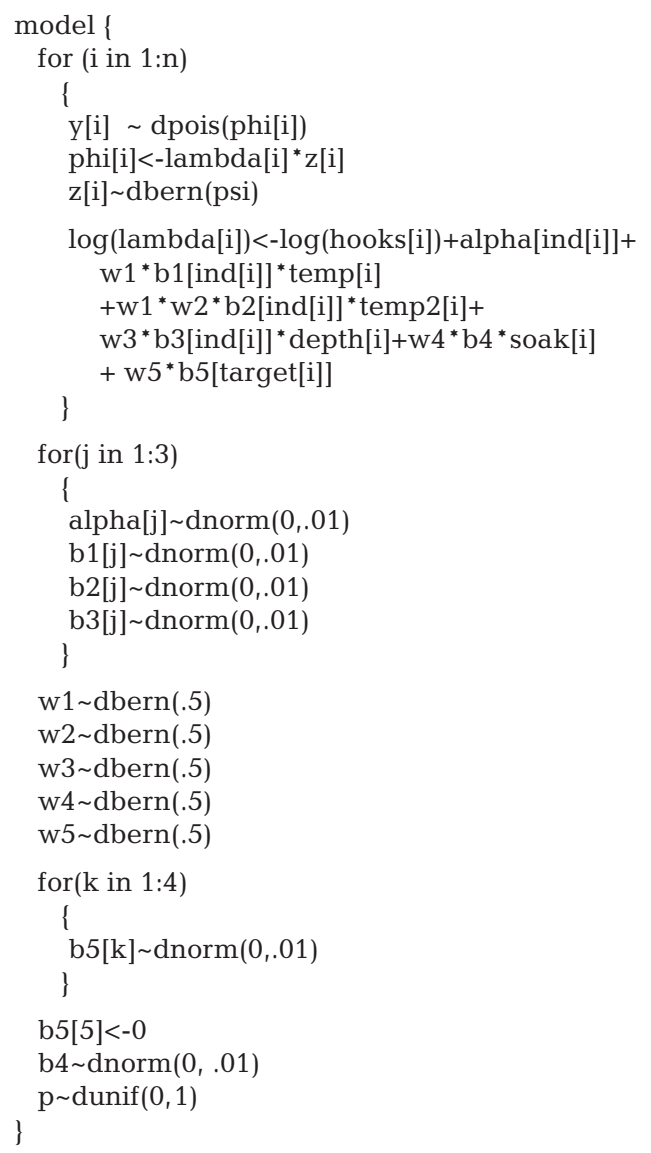




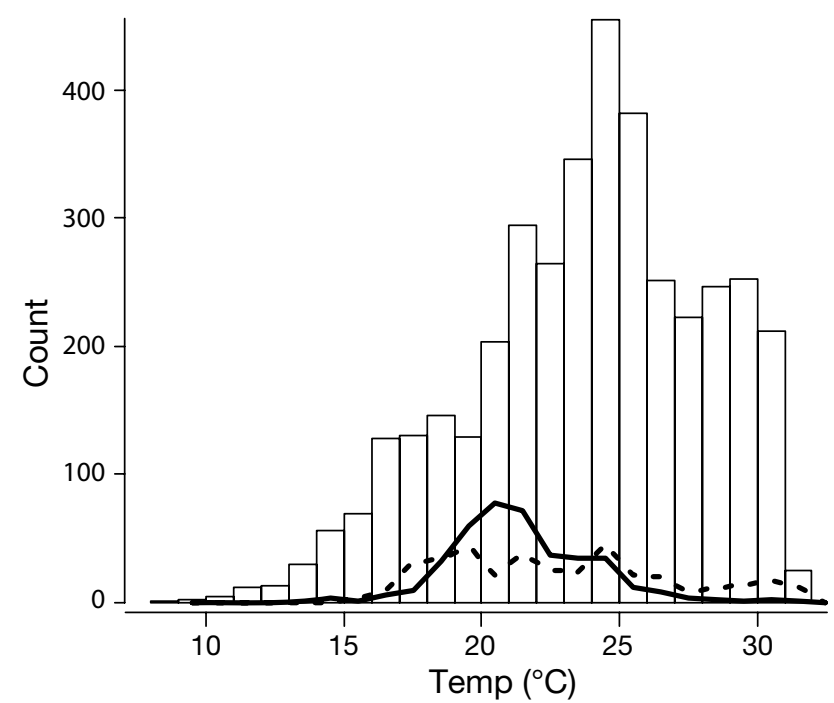

Fig. 2. Temperatures at which fishing occurs across the entire dataset. The $y$-axis is number of fishing sets in each temperature bin $\left(x\right.$-axis in $\left.{ }^{\circ} \mathrm{C}\right)$. Total number of loggerhead (solid line) and leatherback sea turtles (dashed line) caught in each $1^{\circ}$ increment temperature category

tions in the NECMAB display the widest range of temperatures throughout the year. The distribution of fishing across temperatures also varies by region, with a unimodal shape for the NED and NECMAB, and a flat shape across temperatures in the GOM. The most out- standing result that can be seen from Figs. $2 \& 3$ is the high number of sea turtles captured in the NED relative to the amount of fishing (Fig. 3, note the y-axis is different so the total number captured is not as dramatic as it appears). Additionally, Fig. 3 indicates that more leatherback sea turtles were captured in the GOM than loggerhead sea turtles, whereas the NED had more loggerhead sea turtle captures, and the NECMAB showed a similar number of both species.

All of the models achieved convergence based on an evaluation of the MCMC chains from WinBUGS, and all values of Rhat were less than 1.1, which indicates convergence (Gelman \& Hill 2007). However, when using the model extension, we found it was necessary to increase the number of iterations to achieve convergence. In this case, we ran the models for 50000 iterations, and the first 30000 iterations were discarded.

Based on the model selection process, the model with the most weight for the loggerhead sea turtle captures was the one that included temperature and temperature squared, bottom depth, and the target species for the set (Table 1). The posterior summaries for the parameter estimates of the loggerhead sea turtle model are shown in Table 2. Of the target species, the swordfish category had the highest mean value of all the categories (Table 2); however, the credible intervals for swordfish were only significantly different from yellowfin tuna. Although bottom depth was
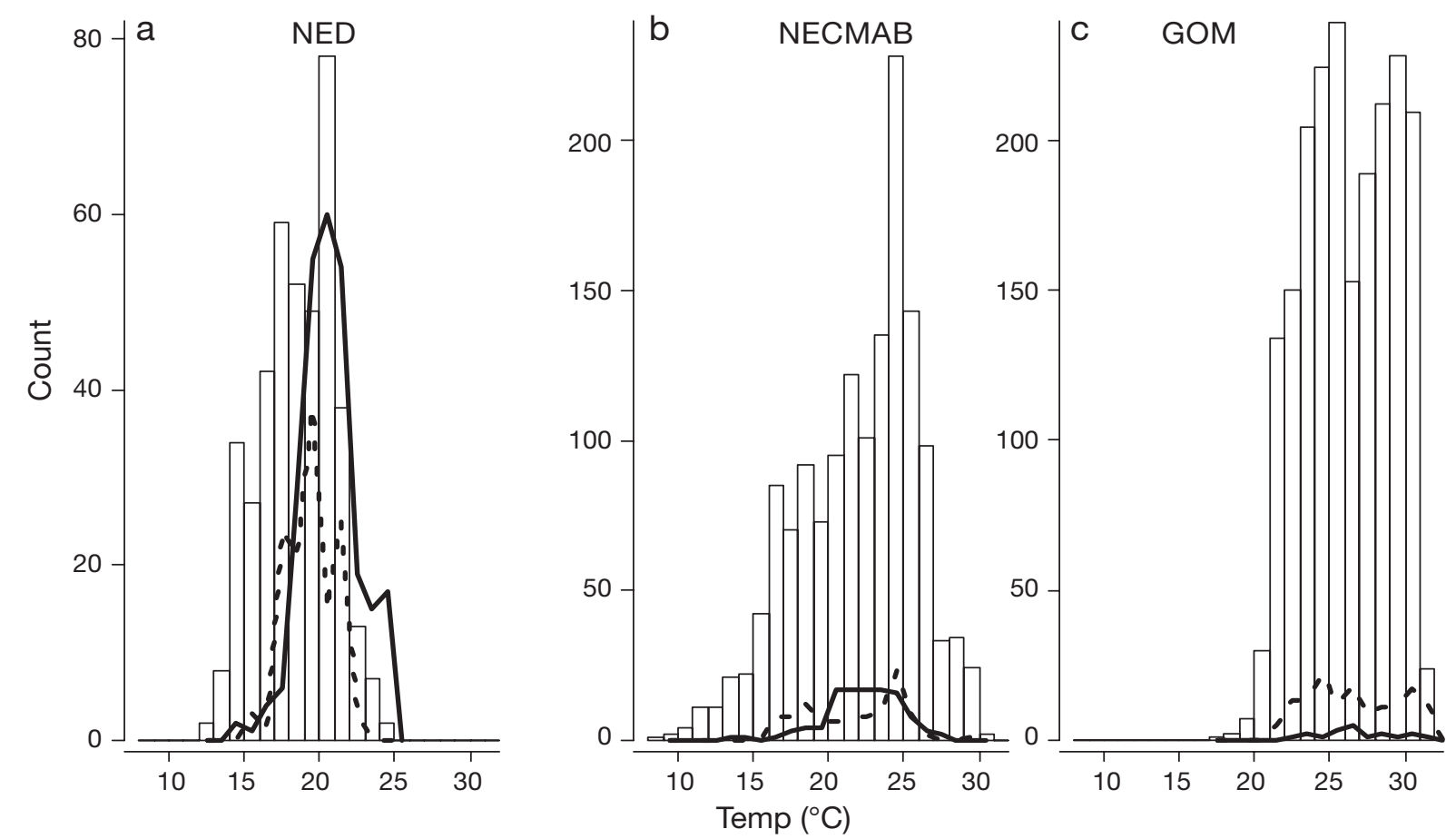

Fig. 3. Temperatures at which fishing occurs by region (a) Northeast Distant (NED), (b) Northeast Coastal combined (NECMAB), (c) Gulf of Mexico (GOM). Bars represent number of fishing sets in a $1^{\circ} \mathrm{C}$ increment category. Total number of loggerhead sea turtles (solid line) and leatherback sea turtles (dashed line) caught in each $1^{\circ} \mathrm{C}$ category are shown. Note the different $y$-axis for (a) 
included in the best model, only the posterior estimate for the NED was significantly greater than 0 . The GOM had the lowest estimated intercept value of loggerhead sea turtle catches of the 3 regions. The conditional effect of temperature and the pattern of the influence of temperature (based on the additive effect of temperature and temperature squared) varied by region (Fig. 4). To show the conditional effect, the other

Table 1. Prior and posterior weights from the model selection process. Selected models are shown, all other models had no posterior weight; 'temp2' refers to both the linear and quadratic temperature effects

\begin{tabular}{|lcl|}
\hline Model & Prior weight & Posterior weight \\
\hline Loggerheads & & \\
null & 0.06 & 0.00 \\
temp2 + depth + target & 0.03 & 0.997 \\
full & 0.03 & 0.003 \\
Leatherbacks & & \\
null & 0.06 & 0.00 \\
target & 0.06 & 0.996 \\
soak + target & 0.06 & 0.004 \\
full & 0.03 & 0.00 \\
\hline
\end{tabular}

covariates in the model were held constant using the mean of each, and we set $\log ($ hooks $)=0$. In the NECMAB, the results show a unimodal curve with the peak number of loggerhead sea turtles captured at $21^{\circ} \mathrm{C}$ (Fig. 4). The results for the NED display a pattern whereby the number of loggerhead sea turtles captured increases almost linearly until $22^{\circ} \mathrm{C}$, at which point the curve starts to decline again (Fig. 4). There was no significant effect of the temperature parameters in the GOM. When the model extension was conducted for the loggerhead sea turtles, the results were very similar. The model with the most weight included the same variables: temperature, the quadratic temperature term, bottom depth, and target species. Due to the similarities in results from both models, we do not present the output from the model extension here.

For the leatherback sea turtles, the model with the most weight included only target species, and a small weight was given to the model with target species and soak time (Table 1); the posterior summaries for the regional means and target species are shown in Table 3. In this model, the temperature variables were not found to be significant and were not assigned any weight in the possible model sets. Sets targeting mixed species and swordfish had the highest estimated means for the number of leatherback sea turtles captured. In both the leatherback and loggerhead sea turtle models, the sets targeted at tuna had a similar posterior mean estimate. As with the loggerhead sea turtle model, the NED had the highest estimated mean number of leatherback sea turtle catches of the 3 regions (based on the intercept term). When the leatherback sea turtle model was modified to allow the zero-inflation parameter to vary by temperature, we found that temperature and temperature squared were included in the best model based on the model selection technique. Specifically, the parameter estimates for the NED and NECMAB were statistically different from 0 based on the $95 \%$ credible interval, while the $95 \%$ credible interval for the parameter estimates of both temperature terms included 0 in the GOM (Table 4). The results for the NED and the NECMAB were similar and displayed a pattern where the probability of catching at least 1 leatherback sea turtle increases until $20^{\circ} \mathrm{C}$, at which point the curve declines (Fig. 5). The probability of catching at least 1 turtle is calculated 


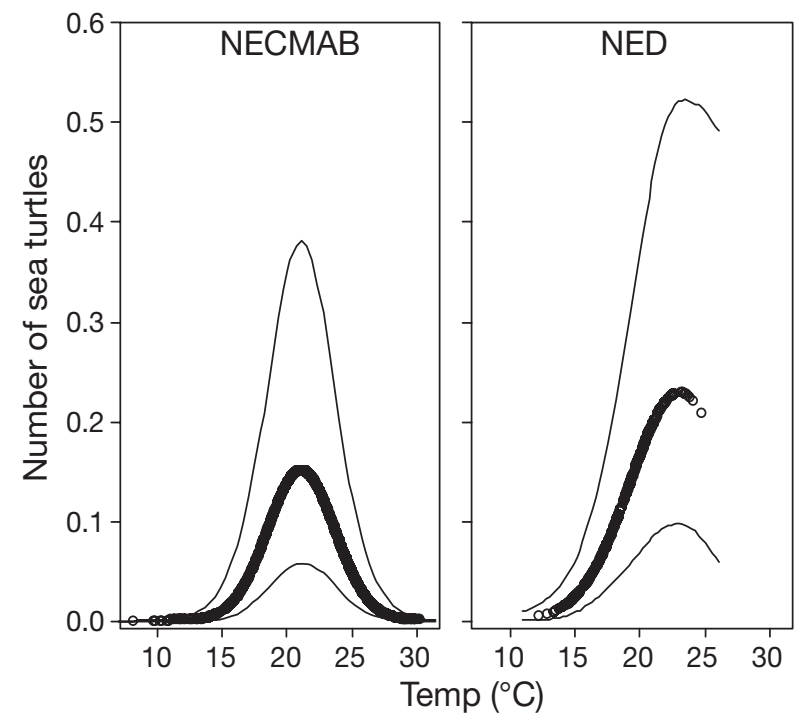

Fig. 4. Conditional effect of temperature (using the linear and quadratic term) for the loggerhead sea turtle model by region-Gulf of Mexico not shown as the parameter estimates for temperature were not significantly different from zero. The $y$-axis is number of loggerhead sea turtles estimated to be captured per set at a given temperature $\left(x\right.$-axis in $\left.{ }^{\circ} \mathrm{C}\right)$. Circles show mean estimates and lines represent $95 \%$ credible interval. NECMAB $=$ Northeast Coastal combined; NED $=$ Northeast distant

as $\psi \times\left(1-e^{-\lambda}\right)$, where $1-\psi$ is the zero-inflation parameter and $e^{-\lambda}$ is the probability of a 0 under the Poisson distribution.

\section{DISCUSSION}

The results of this study show that the relationship between key environmental variables and the incidental capture of sea turtles varies by region. This sug-

Table 3. Posterior summaries of model parameters, given on the log scale, for the leatherback sea turtle model with the most weight (model with only target species). Parameter values are for each region and each target species. Yellowfin tuna is the mean value for the target variable; all the other species values are listed relative to yellowfin tuna. $\mathrm{GOM}=$ Gulf of Mexico, NECMAB $=$ Northeast

Coastal combined, $\mathrm{NED}=$ Northeast distant region, $\mathrm{BET}=$ big-eyed tuna

\begin{tabular}{|llllll|}
\hline Parameter & Mean & SD & $2.5 \%$ & Median & $97.5 \%$ \\
\hline Intercept & & & & & \\
GOM & -2.034 & 0.2254 & -2.498 & -2.029 & -1.61 \\
NECMAB & -2.312 & 0.2706 & -2.862 & -2.305 & -1.797 \\
NED & -0.9576 & 0.305 & -1.574 & -0.9537 & -0.3754 \\
Target & & & & & \\
BET & 1.085 & 0.5213 & 0.004486 & 1.108 & 2.047 \\
Mixed & 1.384 & 0.2354 & 0.9408 & 1.379 & 1.871 \\
Swordfish & 1.232 & 0.278 & 0.6991 & 1.225 & 1.796 \\
Tuna & 0.8048 & 0.2649 & 0.2917 & 0.8025 & 1.335 \\
\hline
\end{tabular}

gests that management decisions aimed at reducing sea turtle bycatch may be more effective if they are spatially explicit. Because sea turtles are poikilotherms, water temperature is generally considered an important variable in analysis. Previous studies (e.g. Watson et al. 2005) have indicated that temperature affects bycatch rates. Our results support that relationship, and we have further quantified how the influence of temperature varies in 3 different regions. To reduce sea turtle captures, regulations could focus on restricting fishing above a specific temperature; however, our study indicates that the relationship between temperature and sea turtle bycatch is not linear in some regions, and thus more regionally specific temperature restrictions could be made. For example, instead of regulating all fishing in waters over $20^{\circ} \mathrm{C}$ to reduce loggerhead sea turtle bycatch in the NECMAB, it might be more effective to regulate fishing in this region in water temperatures between 19 and $24^{\circ} \mathrm{C}$ (where fishing is restricted in water temperatures that have an expected loggerhead sea turtle capture of greater than 0.1). In the GOM, however, due to the lack of a relationship between sea turtle captures and temperature, such restrictions focused on temperature would likely not reduce sea turtle bycatch. This is one example of how management may prove more effective by including a spatially explicit component. It is further possible that other potential variables including gear type, depth of the gear, and bait may also vary by region and thus should be analyzed spatially.

The use of hierarchical models in a Bayesian approach allows for more model flexibility, explicit model assumptions, and a clear model selection technique. Through the zero-inflated Poisson distribution model, we show that the relationship between the number of sea turtles captured and water temperature can be explored in different ways including the number caught as well as the probability of capturing at least 1 sea turtle. Additionally, zero-inflation is often a problem when dealing with rare or elusive species; thus, having a simple model to deal with the large number of zeros should prove useful in many contexts related to bycatch data.

With the loggerhead sea turtle model, we found that all of the variables examined were significant in the model except soak time. The observed differences in the temperature conditions in the 3 regions likely contributed to the results, indicating that the number of sea turtles captured is influenced by the local temperature conditions. The maximum 
Table 4. Posterior summaries of the parameters from the model extension for the leatherback sea turtles taken only from the model with the most weight in the model selection. Temperature and the quadratic temperature term (temperature squared) are given on the logit scale, while all other values are on the log scale. Parameter values are for each region and each target species. Yellowfin tuna is the mean value for the target variable; all the other species values are listed relative to yellowfin tuna. GOM $=$ Gulf of Mexico, NECMAB $=$ Northeast

Coastal combined, NED $=$ Northeast distant region, $\mathrm{BET}=$ big-eyed tuna

\begin{tabular}{|llllll|}
\hline Parameter & Mean & SD & $2.5 \%$ & Median & $97.5 \%$ \\
\hline Intercept & & & & & \\
GOM & -1.961 & 0.2543 & -2.456 & -1.961 & -1.473 \\
NECMAB & -2.139 & 0.3074 & -2.744 & -2.137 & -1.544 \\
NED & -1.009 & 0.3288 & -1.65 & -1.011 & -0.3602 \\
Temperature & & & & & \\
GOM & -0.08216 & 0.3857 & -0.8549 & -0.08132 & 0.6767 \\
NECMAB & -1.467 & 0.3816 & -2.275 & -1.449 & -0.7663 \\
NED & -2.418 & 0.6568 & -3.795 & -2.407 & -1.162 \\
Temperature squared & & & & & \\
GOM & 0.1898 & 0.2993 & -0.3799 & 0.1815 & 0.8031 \\
NECMAB & -0.7988 & 0.2306 & -1.282 & -0.7895 & -0.3796 \\
NED & -1.472 & 0.3571 & -2.234 & -1.46 & -0.8083 \\
Target & & & & & \\
BET & 0.9512 & 0.5272 & -0.1341 & 0.973 & 1.937 \\
Mixed & 1.391 & 0.2421 & 0.9271 & 1.387 & 1.875 \\
Swordfish & 1.19 & 0.2908 & 0.6137 & 1.192 & 1.752 \\
Tuna & 0.8253 & 0.267 & 0.3085 & 0.8251 & 1.357 \\
\hline
\end{tabular}

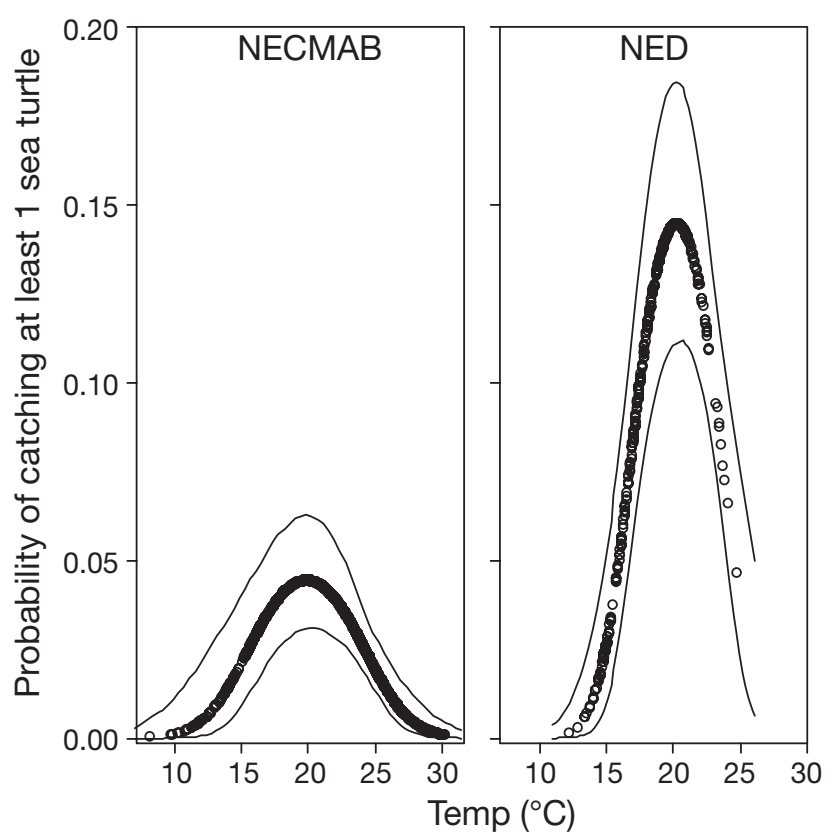

Fig. 5. Conditional effect of temperature (using the linear and quadratic term) for the leatherback sea turtle extended model by region-Gulf of Mexico not shown as parameter estimates for temperature variables were not significantly different from zero. The $y$-axis is the probability of capturing at least 1 leatherback sea turtle per set at a given temperature $\left(x\right.$-axis in $\left.{ }^{\circ} \mathrm{C}\right)$. Circles show mean estimates and lines represent $95 \%$ credible interval. NECMAB = Northeast Coastal combined, NED $=$ Northeast distant region temperature fished in the NED is almost $6^{\circ} \mathrm{C}$ cooler than that of the NECMAB, the adjacent geographical region. In the warmer GOM, where the temperature range is narrower, sea turtles are caught more uniformly across all temperatures. In contrast, the number of loggerhead sea turtles captured in the NECMAB starts to decline at warmer temperatures. The parameter estimates for the linear and quadratic temperature terms varied by region, indicating that the estimated number of loggerhead sea turtles captured at a specific temperature is highly dependent on the region in which fishing is occurring. Arguably, the ecological needs or behavioral activities of sea turtles in different regions may be distinct due to different life stage requirements. If this is the case, management of sea turtles in the different regions may need to be more biologically based to fit the patterns of sea turtle behaviors. The bottom depth parameter was only significant in the NED for the loggerhead sea turtles. Further examination of the data for the NED indicated that this pattern was due primarily to a small number of sets that occurred in deep water with high loggerhead sea turtle catch.

While temperature was not important in predicting the number of leatherback sea turtles, it was an important factor in predicting the zero-inflation parameter. The results suggest that as the temperature increases, the probability of capturing a leatherback sea turtle increases until about 18 to $20^{\circ} \mathrm{C}$, after which it decreases. One possible explanation for this seeming anomaly (that temperature is significant in the model extension but not the original model) is that the number of leatherback sea turtles captured on any given set is often only 1 , and there are few sets with multiple leatherback sea turtle captures (234 sets captured just 1 leatherback sea turtle, while 55 sets captured 2 or more leatherback sea turtles). Aside from the structure of the data, we might expect temperature to be more appropriate for modeling the probability that a turtle will be at the same location as the set based on their probability of spending more time at certain temperatures. On the other hand, target species (a potential proxy for gear type) might influence the number of turtles actually caught, as the depth of the line or bait type, for instance, may affect how many turtles interact with fishing gear. Although leatherback sea turtles do generally have a large thermal mass, they 
still tend to be constrained by preferred temperature ranges (James et al. 2006). In the GOM, we saw no relationship with temperature, but in the NED and NECMAB, we found a unimodal pattern of leatherback sea turtle interactions with the fishery having a peak around $20^{\circ} \mathrm{C}$. This is consistent with other descriptions of the temperature ranges at which sea turtles tend to be most frequently found (Epperly et al. 1995, Polovina et al. 2000).

In addition to temperature, the model estimates show that sets targeting swordfish captured higher numbers of loggerhead sea turtles. Similar results were found for the leatherback sea turtles, as sets targeting mixed species (again, most of these include swordfish among the species being targeted) and swordfish had the highest mean estimates, though not statistically different from the other species except yellowfin tuna. Despite the difference in other variable estimates such as temperature, the 2 sea turtle species had relatively similar estimates for the target species variable, particularly swordfish, thus indicating the importance of targeted species in the number of interactions with sea turtles. Potentially, the target species category is serving as proxy for gear type or another variable and thus further investigation would be warranted to help reduce the number of interactions between the fishery and sea turtles.

Modeling sea turtle bycatch data can be difficult since the data often represent a rare event and the count data include a large number of zeros (McCracken 2004). Techniques to account for such data include zero-inflated models (e.g. the Poisson, ZIP; or negative-binomial, ZINB) and delta-F methods (see Martin et al. 2005 or Minami et al. 2007 for a comparison of the techniques). Minami et al. (2007) suggest that ZIP models are more appropriate when a species is caught infrequently but when present are in smaller groups, whereas the ZINB is appropriate when a species is caught infrequently but are present in larger groups when caught. Lennert-Cody et al. (2004) use a ZIP model for analyzing dolphin bycatch, while Minami et al. (2007) use a ZINB for shark bycatch. Sea turtle species, when captured in the Atlantic pelagic longline fishery, tend to appear alone or in smaller groups (generally, 1 to 3 turtles per set) and thus a ZIP model seemed to be appropriate for such data.

The hierarchical ZIP modeling approach we took allows for incorporating other useful extensions to the model. For example, adding covariate information into the model for $\mathrm{E}\left[z_{i}\right]$ permits covariates to directly influence the probability that a sea turtle is captured, not just the number captured. Also, because of the model structure, one could examine covariates in relation to the zero-inflation parameter and the mean of the Poisson distribution simultaneously. It is not clear how this could be done using a non-hierarchical approach. Certain biological mechanisms, for example clustering or inhibition in species distributions, might make one analysis more insightful over the other. Another model extension would be adjusting the WinBUGS model specification to define the parameters as random effects instead of fixed effects. This simple model extension would allow for the parameter estimates to vary by region (or by any category of a given variable) but with an overriding distribution on the parameter. In our application, there were only 3 regions of interest and 5 target species categories; however, in a study looking at more regions, more species, or more target species categories, specifying the coefficients as random effect parameters would provide a concise mechanism for examining the potential interactions between parameters (e.g. the interaction between region and temperature). Gelman \& Hill (2007) provide a concise but informative discussion on random and fixed effects, including the number of categories and use of the terms 'fixed' and 'random'.

James et al. (2005) suggest that for conservation of sea turtles in the pelagic waters of the Atlantic, modifying fishing practices may be more effective than implementing area closures. In fact, in 2004, gear modifications were required of the US fishermen in the Atlantic in order to reduce sea turtle interactions (US Department of Commerce 2004). Our study examined data from 1992 to 2003, which can be used in future years for comparing the probability of capture or number of sea turtles captured after implementation of the gear modifications. Noting the gear modification impacts across regions and temperature ranges will be insightful into the effectiveness of this type of management practice. Thus, the results of this study, showing the impact of spatially explicit temperature-dependence on the number of sea turtles caught, are useful as a practical application for management now and as a monitoring tool for future assessment of fishery management effectiveness.

Rules or regulations, such as not allowing fishing above a certain temperature over wide expanses of an ocean basin, are likely too broad to be effective for reducing sea turtle catches without negatively impacting the fishing industry. The ability to refine management actions based on the interactions of spatial units (e.g. regions) and environmental variables could prove to be as effective as altering fishing practices. The Bayesian approach described here provides a useful framework for future refinements to modeling fisheries interactions with sea turtles. The framework provides a concise and explicit mechanism for examining variable interactions, model selection, and other distributions (e.g. binomial, poisson, negative-binomial), and can be employed in future management strategies for the pelagic longline fishery. 
Acknowledgements. The authors thank the Southeast Fisheries Science Center for providing the Pelagic Observer Program data and the National Marine Fisheries Service and Sea Grant for funding. Thanks also to Steve DeGloria, Robert Strawderman, J. Andrew Royle, Eduardo Carrillo-Rubio, David Hodgson and 2 anonymous reviewers for comments on this manuscript.

\section{LITERATURE CITED}

Beerkircher LR, Brown CJ, Abercrombie DL, Lee DW (2004) SEFSC pelagic observer program data summary for 1992-2002. NOAA Tech Memo NMFS-SEFSC-522. Available at: http://www.sefsc.noaa.gov/PDFdocs/newlett_02_ proof.pdf

Coles WC, Musick JA (2000) Satellite sea surface temperature analysis and correlation with sea turtle distribution off North Carolina. Copeia 2000:551-554

Congdon P (2007) Model weights for model choice and averaging. Stat Methodol 4:143-157

Dobson AJ (1990) An introduction to generalised linear models. Chapman \& Hall, London

Epperly SP, Braun J, Chester AJ, Cross FA, Merriner JV, Tester PA (1995) Winter distribution of sea turtles in the vicinity of Cape Hatteras and their interactions with the summer flounder trawl fishery. Bull Mar Sci 56:547-568

Frair W, Ackman RG, Mrosovsky N (1972) Body temperature of Dermochelys coriacea: warm turtle from cold water. Science 177:791-793

Gelman A (2003) Bugs.R: functions for running WinBUGS from R. Available at: www.stat.columbia.edu/ gelman/ bugsR/

Gelman A, Hill J (2007) Data analysis using regression and multilevel/hierarchical models. Cambridge University Press, New York

Gilman E, Zollett E, Beverly S, Nakano H and others (2006) Reducing sea turtle by-catch in pelagic longline fisheries. Fish Fish 7:2-23

Gilman E, Kobayashi D, Swenarton T, Brothers N, Dalzell P, Kinan-Kelly I (2007) Reducing sea turtle interactions in the Hawaii-based longline swordfish fishery. Biol Conserv 139:19-28

IUCN (International Union for Conservation of Nature and Natural Resources) (2004) IUCN redlist of threatened species. Cambridge. Available at: www.redlist.org/ (accessed October 2006; updated October 2006)

- James MC, Ottensmeyer CA, Myers RA (2005) Identification of high-use habitat and threats to leatherback sea turtles in northern waters: new directions for conservation. Ecol Lett 8:195-201

James MC, Sherrill-Mix SA, Martin K, Myers RA (2006) Canadian waters provide critical foraging habitat for leatherback sea turtles. Biol Conserv 133:347-357

Kobayashi DR, Polovina JJ (2005) Evaluation of time-area closures to reduce incidental sea turtle take in the Hawaiibased longline fishery: generalized additive model (GAM) development and retrospective examination. NOAA Tech Memo NMFS-PIFSC-4. Available at: www.pifsc. noaa.gov/tech/NOAA_Tech_Memo_PIFSC_4.pdf

Kuo L, Mallick B (1998) Variable selection for regression models. Sankhya Ser B 60:65-81

Lennert-Cody CE, Minami M, Hall MA (2004) Incidental mortality of dolphins in the eastern Pacific Ocean purseseine fishery: correlates and their spatial association. J Cetacean Res Manag 6:151-163

> Lewison RL, Crowder LB (2007) Putting longline bycatch of sea turtles into perspective. Conserv Biol 21:79-86

Lewison RL, Freeman SA, Crowder LB (2004) Quantifying the effects of fisheries on threatened species: the impact of pelagic longlines on loggerhead and leatherback sea turtles. Ecol Lett 7:221-231

Link WA, Barker RJ (2006) Model weights and the foundations of multimodel inference. Ecology 87:2626-2635

- Martin TG, Wintle BA, Rhodes JR, Kuhnert PM and others (2005) Zero tolerance ecology: improving ecological inference by modelling the source of zero observations. Ecol Lett 8:1235-1246

McCracken M (2004) Modelling a very rare event to estimate sea turtle bycatch. NOAA Tech Memo NMFSPIFSC-3. Available at: www.pifsc.noaa.gov/tech/NOAA_ Tech_Memo_PIFSC_3.pdf

Minami M, Lennert-Cody CE, Gao W, Roman-Verdesoto M (2007) Modeling shark bycatch: the zero-inflated negative binomial regression model with smoothing. Fish Res 84: 210-221

NMFS (National Marine Fisheries Service Southeast Fisheries Science Center) (2001) Stock assessments of loggerhead and leatherback sea turtles and an assessment of the pelagic longline fishery on the loggerhead and leatherback sea turtles of the Western North Atlantic. NOAA Tech Memo NMFS-SEFSC-455. Available at: www.sefsc.noaa.gov/PDFdocs/TM_455_NMFS.pdf

Paladino FV, O'Connor MP, Spotila JR (1990) Metabolism of leatherback turtles, gigantothermy, and thermoregulation of dinosaurs. Nature 344:858-860

Polovina JJ, Kobayashi DR, Parker DM, Seki MP, Balazs GH (2000) Turtles on the edge: movement of loggerhead turtles (Caretta caretta) along oceanic fronts, spanning longline fishing grounds in the central North Pacific, 1997-1998. Fish Oceanogr 9:71-82

Royle JA, Dorazio R (2008) Hierarchical modeling and inference in ecology: the analysis of data from populations, metapopulations and communities. Academic Press, San Diego, CA

Shoop CR, Kenney RD (1992) Seasonal distributions and abundances of loggerhead and leatherback sea turtles in waters of the northeastern United Sates. Herpetol Monogr 6:43-67

US Department of Commerce (2004) Atlantic highly migratory species (HMS); pelagic longline fishery. Fed Regist 69:40734-40758

Watson JW, Epperly SP, Shah AK, Foster DG (2005) Fishing methods to reduce sea turtle mortality associated with pelagic longlines. Can J Fish Aquat Sci 62:965-981

Williams P, Annions PJ, Plotkin PT, Salvini KL (compilers) (1996) Pelagic longline fishery-sea turtle interactions: proceedings of an industry, academic and government experts, and stakeholders workshop held in Silver Spring, Maryland, 24-25 May 1994. NOAA Tech Memo NMFS-OPR-7. Available at: www.sefsc.noaa.gov/ PDFdocs/TM_NMFS_OPR_7_Williams_etal.pdf

Witzell WN (1999) Distribution and relative abundance of sea turtles caught incidentally by the US pelagic longline fleet in the western North Atlantic Ocean, 1992-1995. Fish Bull (Wash DC) 97:200-211 